\title{
NOTES
}

\section{Orientation Study of Poly(ethylene terephthlate) Ultrathin Films during Crystallization}

\author{
Ying ZHANG, Yonglai Lu, Shouke YAN, and Deyan SHEN ${ }^{\dagger}$ \\ State Key Laboratory of Polymer Physics and Chemistry, Joint Laboratory of Polymer Science and Material, \\ Institute of Chemistry, Chinese Academy of Sciences, Beijing 100080, People's Republic of China
}

(Received August 16, 2004; Accepted November 24, 2004; Published February 15, 2005)

KEY WORDS Crystallization Orientation / Poly(ethylene terephthlate) / Ultrathin Films / [DOI 10.1295/polymj.37.133]

In recent years there are numerous applications and processes in which ultrathin polymer films $(<$ about $100 \mathrm{~nm}$ ) can be found. The role of confinement and interface in altering the physical behavior of polymers has also drawn more attention. It has been found that depending on the thickness of ultrathin films, the glass transition temperature, ${ }^{1-6}$ crystallization behavior, ${ }^{7-9}$ morphology ${ }^{10,11}$ and electrical properties, ${ }^{12}$ etc. may be altered. In addition, the polymeric systems in ultrathin films usually show preferred orientation different from that of bulks. For example, Frank et al. ${ }^{8,9}$ disclosed that while the backbones of poly(di- $n$-hexylsilane) deposited on the quartz are oriented preferentially in the plane of the film, the mean orientation of poly(ethylene oxides) molecules on oxidized silicon substrate is in the surface-normal direction. Moreover, the PEO lamellae showed interesting film thickness dependent morphology and orientation. Similar study of orientation in ultrathin films can also be found in other polymeric systems. ${ }^{13-16}$

Variety kinds of method ${ }^{8,9,13-16}$ can be used to study the orientation of polymer in the confined geometry. Among them, Fourier transform infrared (FT-IR) spectroscopy is a usefully technique and has great advantage: it can probe directly to such subtle details as intermolecular interactions, localized molecular conformations and orientation. As one of various kinds of infrared techniques, reflection-absorption infrared (RAIR) spectroscopy is well fit to the characterization of the ultrathin films with thickness of nanometer level. The characteristic ${ }^{17}$ of RAIR is that the resultant electric field vector is perpendicular to the metal surface. Therefore, if molecules are adsorbed onto the substrate with a preferred orientation, vibration modes having transition moments perpendicular to the surface will appear with greater intensity than modes having transition moments parallel to the sur- face. So RAIR is especially useful for determining the orientation of adsorbed molecular species. In this work, the orientation of poly(ethylene terephthlate) (PET) in ultrathin films was determined by the combination of RAIR and IR transmission. As a kind of polymer with considerable commercial importance, the property of PET in bulk has been extensively studied. But the corresponding report of its ultrathin film cannot be found by far. The thickness dependence of the orientation during isothermal crystallization was also investigated.

\section{EXPERIMENTAL}

\section{Sample Preparation}

PET pellets $\left(M_{\mathrm{w}} \sim 36000-41000 \mathrm{~g} / \mathrm{mol}, M_{\mathrm{w}} / M_{\mathrm{n}} \sim\right.$ 1.8-2.05) were produced by Changzheng Chemical Industries, Beijing. The PET pellets were dissolved in chloroform-trifluoroacetic acid $(9: 1(\mathrm{v} / \mathrm{v}))$. All glass wafers were cleaned by fresh piranha solution $\left(30 \% \mathrm{H}_{2} \mathrm{O}_{2}\right.$ mixed in 1:5 ratio with concentrated $\mathrm{H}_{2} \mathrm{SO}_{4}$ ). The wafers were then rinsed with copious quantities of deionized water, and dried under a warm nitrogen flow. A $20 \mathrm{~nm}$ adhesion layer of chromium and $110 \mathrm{~nm}$ layer of $\mathrm{Au}$ were deposited on the glass wafers by thermal evaporation in a vacuum chamber (HITACHI HUS-5GB). The vacuum is $10^{-5}-10^{-6}$ Torr. The ultrathin films of different thicknesses were prepared by spin-coating PET solutions of various concentrations at a speed of $2000 \mathrm{rpm}$ for about $60 \mathrm{~s}$ onto the gold-coated glass wafers. The films were kept under vacuum at ambient temperature for $2 \mathrm{~d}$ to remove the residual solvent. Before measurement, all samples were annealed at about $85^{\circ} \mathrm{C}$ for $4 \mathrm{~h}$ and then slowly cooled to room temperature. The films after annealing at $85^{\circ} \mathrm{C}$ for $4 \mathrm{~h}$ were amorphous because the fraction of trans conformer is nearly $10 \% .^{18-20}$

${ }^{\dagger}$ To whom correspondence should be addressed (E-mail: dyshen@iccas.ac.cn). 


\section{Thickness Determination}

Thickness measurements of thin films were performed with NanoScope IIIA MultiMode atomic force microscope (AFM) (Digital Instrument) in tapping mode. The film thickness was determined by AFM height profile after partially removing the thin film from the glass wafers. The thickness of gold film and PET-gold film were measured respectively. They were defined as the distance between the glass surface and the average of all height values taken in a line scan across the sample surface. ${ }^{21}$ To minimize the experimental errors, several height profiles taken from different location of one sample were averaged. Then the thickness of PET thin film was acquired by subtracting the gold film thickness from that of PET-gold film. Considering the absorbance of RAIR spectra increase almost linearly with thickness for thin films having thickness less than about $400 \mathrm{~nm},{ }^{22}$ Most of the film thicknesses were deduced by comparing their intensities of $1410 \mathrm{~cm}^{-1}$ band in IR spectra, which is related to thickness variations, ${ }^{23}$ with several AFMmeasured thicknesses of the films.

\section{Reflection-Absorption FT-IR Measurement}

Reflection-absorption FT-IR spectra in the region of $400-4000 \mathrm{~cm}^{-1}$ were collected with a Bruker EQUINOX 55 FT-IR spectrometer equipped with an MCT detector. The measurements were obtained by average 32 scans and at a resolution of $4 \mathrm{~cm}^{-1}$. The incidence angle was fixed at $83^{\circ}$ for the best signal recording. The polarization of the incoming beam was parallel to the plane of incidence (p-polarized). A homemade heating stage was used to obtain in-situ FT-IR spectra. The temperature was controlled to $\pm 0.3^{\circ} \mathrm{C}$. For isothermal crystallization, the prepared amorphous films were put onto the heating stage at a predetermined temperature and were kept there for certain times after equilibrium was reached.

\section{RESULTS AND DISCUSSION}

The preferred orientation of the main-chain of PET molecule in ultrathin film on gold substrate was interrogated by comparing the transmission mode and (p-polarized) RAIR spectrum. Shown in Figure 1 are the transmission spectra of PET film coated on a $\mathrm{KBr}$ pellet and the RAIR spectra of a $43 \mathrm{~nm}$ PET ultrathin film spin-coated on gold surface. Both films we used are amorphous. Figure $1 \mathrm{a}$ and $\mathrm{b}$ give the spectra of the different wavenumber region in which the bands we studied are evident. It is known that reflectance spectra clearly show large changes in both peak position and shape compared to transmission spectra. ${ }^{24,25}$ The phenomenon is indeed observed from the band shift of $\mathrm{C}=\mathrm{O}$. To get the qualitative informa-
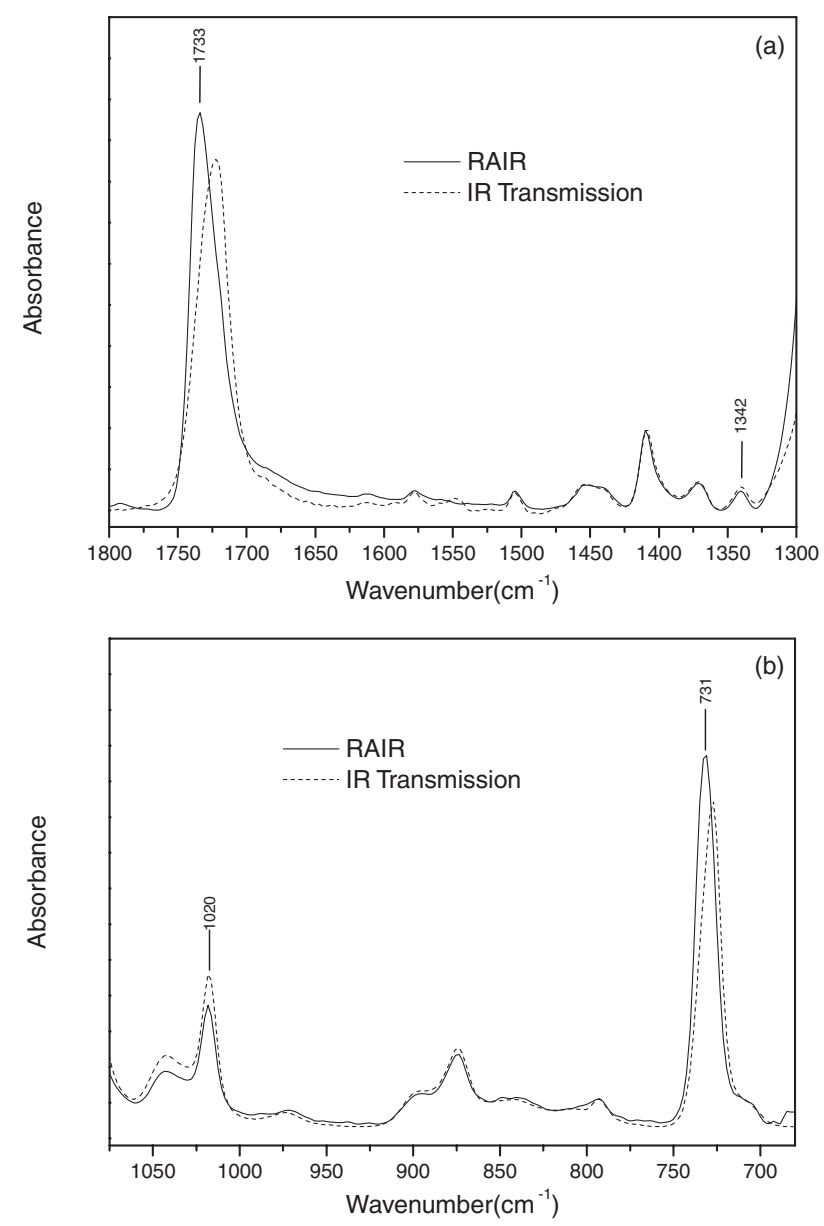

Figure 1. RAIR spectrum of a $43 \mathrm{~nm}$ PET film spin-coated on gold and IR transmission spectrum of PET film coated on the $\mathrm{KBr}$ pellet, (a) and (b) are in different wavenumber region.

tion about the molecular orientation, we avoid the disturbance of the band distortions by comparing the relative intensity of band in the same spectra.

When comparing with IR transmission spectra, the differences appear in the RAIR spectra of PET ultrathin films. In Figure 1a, the intensity of $1733 \mathrm{~cm}^{-1}$ band exhibits increase relative to that of band in $1342 \mathrm{~cm}^{-1}$. As are summarized in Table I, the bands at 1733 and $1342 \mathrm{~cm}^{-1}$ are assigned to $\mathrm{C}=\mathrm{O}$ stretching and $\mathrm{CH}_{2}$ wagging vibration, and their transition moments are perpendicular and parallel to the backbone of PET, respectively. ${ }^{26,27}$ The similar relative intensity changes are also shown in Figure 1b. The band near $1020 \mathrm{~cm}^{-1}$ that is assigned to in-plane bending vibration of ring $\mathrm{CH}$ is parallel band, and the combination band of out-of-plane bending vibration for $\mathrm{C}=$ $\mathrm{O}$ and ring $\mathrm{CH}$ at $731 \mathrm{~cm}^{-1}$ is perpendicular band. ${ }^{26,27}$ The ratio of the intensity of $731 \mathrm{~cm}^{-1}$ relative to that of the parallel band at $1020 \mathrm{~cm}^{-1}$ also increased. This indicates that there is a selective orientation for the PET backbone on the gold substrate. On the basis of the mutually perpendicular direction of the electric field vector for transmission vs. p-polarized RAIR 
Table I. Band assignment and polarization of IR spectrum of PET

\begin{tabular}{cccc}
\hline $\begin{array}{c}\text { Wavenumber } \\
\left(\mathrm{cm}^{-1}\right)^{\mathrm{a}}\end{array}$ & $\begin{array}{c}\text { Relative } \\
\text { intensity }\end{array}$ & $\begin{array}{l}\text { Band assignment } \\
\text { of the band } \\
\text { discussed }^{\mathrm{c}}\end{array}$ & Polarization $^{\mathrm{d}}$ \\
\hline $\begin{array}{c}1733(1724) \\
1370\end{array}$ & $\mathrm{vs}$ & $\mathrm{v}(\mathrm{C}=\mathrm{O})$ & $\perp, \pi$ \\
1342 & $\mathrm{~m}$ & $\begin{array}{c}\mathrm{wag}\left(\mathrm{CH}_{2}\right) \\
\text { wag }\left(\mathrm{CH}_{2}\right)\end{array}$ & $\|$ \\
1020 & $\mathrm{~m}$ & $\begin{array}{c}\beta(\mathrm{CH} \text { of phenyl } \\
\text { ring })\end{array}$ & $\|$ \\
$731(727)$ & $\mathrm{s}$ & $\begin{array}{c}\gamma(\mathrm{C}=\mathrm{O})+\gamma \\
(\mathrm{CH} \text { of phenyl } \\
\text { ring })\end{array}$ & $\perp, \sigma$ \\
\hline
\end{tabular}

${ }^{\mathrm{a}}$ The wavenumbers in bracket represent the peak positions in transmission IR spectra. ${ }^{\mathrm{b}} \mathrm{s}=$ strong, $\mathrm{w}=$ weak, $\mathrm{m}=$ medium, vs $=$ very strong ${ }^{c} v=$ stretching, wag $=$ wagging, $\beta=$ inplane bending. $\gamma=$ out-of-plane bending. ${ }^{\mathrm{d}} \perp$ and $\|$ denote perpendicular and parallel polarization, respectively, with respect to the main-chain axis of PET molecules. $\pi$ and $\sigma$ denote parallel and perpendicular to the plane of the phenyl ring respectively.

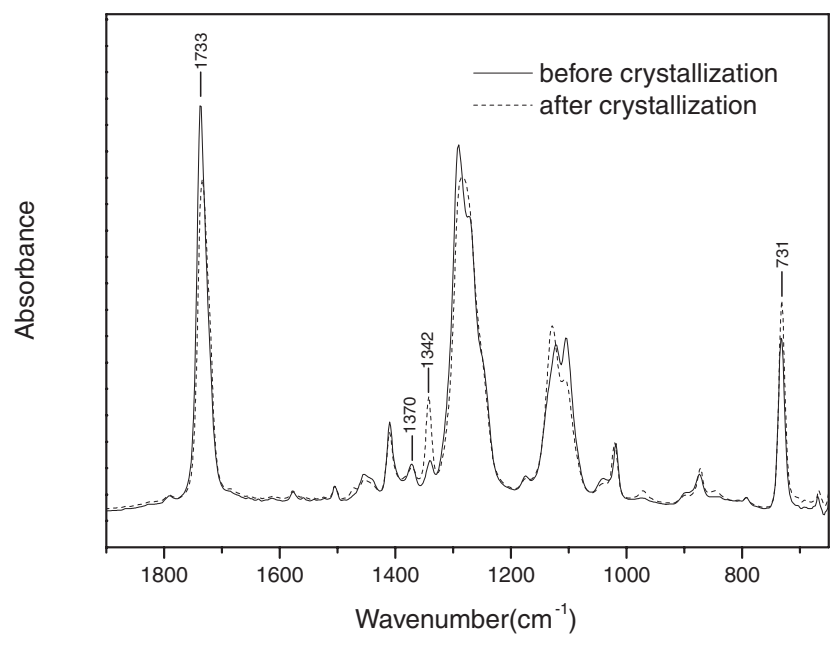

Figure 2. RAIR spectra of a $115 \mathrm{~nm}$ film before and after isothermal crystallization at $110^{\circ} \mathrm{C}$.

modes, we can concluded that the main-chain of PET in ultrathin film shows some alignment parallel to the substrate. It is believed that the chain orientation of the PET ultrathin film should be caused by the sample preparation method and substrate property.

The orientation during crystallization of the PET ultrathin film was also investigated. Figure 2 gives the RAIR spectrum of a $115 \mathrm{~nm}$ PET film crystallized isothermally at $110^{\circ} \mathrm{C}$. The spectrum of uncrystallized film with the same thickness is also given for contrast. It can be found from Figure 2 that the two bands 1733 and $731 \mathrm{~cm}^{-1}$ display difference in the relative intensities. After crystallization, the intensity of $1733 \mathrm{~cm}^{-1}$ band decreased while that of $731 \mathrm{~cm}^{-1}$ band increased. As mentioned above, these two bands are both per-
Table II. Values of $A_{731} / A_{1733}$ and crystallinity $T_{\mathrm{c}}$ of PET ultrathin films isothermal crystallized at 100,105 and $110^{\circ} \mathrm{C}$

\begin{tabular}{cccc}
\hline $\begin{array}{l}\text { Crystallization } \\
\text { temperature }\left({ }^{\circ} \mathrm{C}\right)\end{array}$ & $\begin{array}{c}\text { Film thickness } \\
(\mathrm{nm})\end{array}$ & $A_{731} / A_{1733}$ & $\begin{array}{c}\text { Crystallinity } \\
T_{\mathrm{c}}\end{array}$ \\
\hline \multirow{3}{*}{100} & 22 & 0.444 & 0.260 \\
& 40 & 0.449 & 0.344 \\
& 58 & 0.498 & 0.375 \\
& 102 & 0.520 & 0.382 \\
\hline \multirow{3}{*}{105} & 22 & 0.430 & 0.267 \\
& 41 & 0.467 & 0.366 \\
& 66 & 0.491 & 0.399 \\
& 109 & 0.552 & 0.424 \\
\hline \multirow{3}{*}{110} & 22 & 0.433 & 0.306 \\
& 43 & 0.488 & 0.375 \\
& 67 & 0.494 & 0.449 \\
& 115 & 0.640 & 0.455 \\
\hline
\end{tabular}

pendicular polarization. But the transition moment of $1733 \mathrm{~cm}^{-1}$ band is parallel to the plane of phenyl ring while it is perpendicular to the plane for the transition moment of $731 \mathrm{~cm}^{-1}$ band. ${ }^{26,28}$ Therefore, from the relative intensities change of these two bands it can be concluded that during crystallization both the phenyl ring and carbonyl group in PET molecular chain tend to orient more parallel to substrate. Furthermore, the orientation is found different with varying film thickness. The effect of film thickness on the orientation was obtained more quantitatively by analyzing the intensity ratio of these two bands. Table II gives the intensity ratios $A_{731} / A_{1733}$ of films with various thickness when the isothermal crystallization at 100, 105 and $110^{\circ} \mathrm{C}$ are completed. It indicates that in each crystallization temperature the value of $A_{731} / A_{1733}$ displays an evidently increasing trend with film thickness.

The corresponding crystallinities $T_{\mathrm{c}} \mathrm{s}$ of the films at all crystallization temperatures are also shown in Table II. The crystallinity can be determined from the fraction of trans conformers of PET. ${ }^{18,20,29,30} \mathrm{We}$ choose the two bands 1342 and $1370 \mathrm{~cm}^{-1}$ as key bands for determining the relative conformational population. These two bands have been assigned to the $\mathrm{CH}_{2}$ (in $-\mathrm{O}-\mathrm{CH}_{2}-\mathrm{CH}_{2}-\mathrm{O}-$ moiety) wagging mode in trans and gauche conformers, respectively. ${ }^{26}$ It can be found in Table II that the calculated $T_{\mathrm{c}} \mathrm{S}$ also show significant thickness dependence, which is interestingly consistent with those of $A_{731} / A_{1733}$. In our other study, ${ }^{31}$ the isothermal and non-isothermal crystallization kinetics of PET ultrathin films with different thickness were investigated. It was found that during isothermal crystallization the thinner PET film shows slower kinetic than the thicker film. Moreover, the obtained Avrami exponents decreased with the film thickness. As for the case of the nonisothermal crystallization, the cold-crystallization starting tempera- 
ture shifted to lower temperature as film thickness increased. It is believed that the confinement effect contributes to the above experimental results. In a confined geometry such as in ultrathin film the growth of the crystalline may be inhibited by the film thickness. This confinement effect is more significant in thinner film. The thickness dependences of orientation and crystallinity in our study may serve as evidences of existence of this confinement effect.

In conclusion, the orientation of PET ultrathin films was investigated by the combination of RAIR and IR transmission. It is concluded that the main-chain of PET molecule in ultrathin film is prone to alignment parallel to the gold substrate. During isothermal crystallization, the phenyl and $\mathrm{C}=\mathrm{O}$ in molecule chain of PET tended to take orientation more parallel to the substrate. Moreover, the orientation also showed significant thickness dependence. This was consistent with thickness dependence of the crystallinity during isothermal crystallization.

Acknowledgment. This work was financially supported by the Chinese Academy of Sciences and the National Natural Science Foundation of China.

\section{REFERENCES}

1. J. L. Keddie, R. A. L. Jones, and R. A. Cory, Faraday Discuss., 98, 219 (1994).

2. O. K. C. Tsui, T. P. Russell, and C. J. Hawker, Macromolecules, 34, 5535 (2001).

3. D. S. Fryer, R. D. Peters, E. J. Kim, J. E. Tomaszewski, J. J. D. Pablo, P. F. Nealey, C. C. White, and W. L. Wu, Macromolecules, 34, 5627 (2001).

4. G. B. Demaggio, W. E. Frieze, D. W. Gidley, M. Zhu, H. A. Hristov, and A. F. Yee, Phys. Rev. Lett., 78, 1524 (1997).

5. J. A. Forrest, K. Dalnoki-Veress, J. R. Stevens, and J. R. Dutcher, Phys. Rev. Lett., 77, 2002 (1996).

6. Y. Zhang, J. Zhang, Y. Lu, Y. Duan, S. Yan, and D. Shen, Macromolecules, 37, 2532 (2004).

7. M. M. Despotopoulou, C. W. Frank, R. D. Miller, and J. F. Rabolt, Macromolecules, 29, 5797 (1996).

8. M. M. Despotopoulou, R. D. Miller, J. F. Rabolt, and C. W. Frank, J. Polym. Sci., Part B: Polym. Phys., 34, 2335 (1996).
9. H. Schonherr and C. W. Frank, Macromolecules, 36, 1188 (2003).

10. M. J. Fasolka, P. Banerjee, A. M. Mayes, G. Pickett, and A. C. Balazs, Macromolecules, 33, 5701 (2000).

11. S. J. Sutton, K. Izumi, H. Miyaji, Y. Miyamoto, and S. Miyatashi, J. Mater. Sci., 32, 5621 (1997).

12. T. Liang, Y. Makita, and S. Kimura, Polymer, 42, 4867 (2001).

13. Z. Bartczak, A. S. Argon, R. E. Cohen, and T. Kowalewski, Polymer, 40, 2367 (1999).

14. C. L. Hoffman and J. F. Rabolt, Macromolecules, 29, 2543 (1996).

15. O. K. Muratoglu, A. S. Argon, and R. E. Cohen, Polymer, 36, 2143 (1995).

16. G. D. Hietpas and D. L. Allara, J. Polym. Sci., Part B: Polym. Phys., 36, 1247 (1998).

17. S. A. Francis and A. H. Ellison, J. Opt. Soc. Am., 49, 131 (1959).

18. R. Qian, D. Shen, and F. Sun, Macromol. Chem. Phys., 197, 1485 (1996).

19. S. Lin and J. Koenig, J. Polym. Sci., Polym. Phys. Ed., 20, 2277 (1982).

20. S. Lin and J. Koenig, J. Polym. Sci., Polym. Phys. Ed., 21, 2365 (1983).

21. J. Kressler, C. Wang, and H. W. Kammer, Langmuir, 13, 4407 (1997).

22. F. J. Boerio, J. T. Young, and W. W. Zhao, in "Multidimensional Spectroscopy of Polymers: Vibrational, NMR, and Fluorescence Techniques," M. W. Urban and T. Provder, Ed., American Chemical Society, Washington, DC, 1995, chapt. 2, p 8.

23. V. M. De Cupere and P. G. Rouxhet, Polymer, 43, 5571 (2002).

24. D. L. Allara, A. Baca, and C. A. Pryde, Macromolecules, 14, 1215 (1978).

25. B. Schneider, J. Stokr, P. Schmidt, M. Mihajlov, S. Dirlikov, and N. Peeva, Polymer, 20, 705 (1979).

26. A. Miyake, J. Polym. Sci., 38, 479 (1959).

27. C. Y. Liang and S. Krimm, J. Mol. Spectrosc., 3, 554 (1959).

28. F. J. Boerio and S. K. Bahl, J. Polym. Sci., Polym. Phys. Ed., 14, 1029 (1976).

29. P. G. Schmidt, J. Polym. Sci., Part A: Regul. Pap. 1, 1271 (1963).

30. W. Zhang and D. Shen, Polym. J., 30, 311 (1998).

31. Y. Zhang, Y. Lu, Y. Duan, J. Zhang, S. Yan, and D. Shen, J. Polym. Sci., Polym. Phys. Ed., 42, 4440 (2004). 